\title{
Theoretical PAH emission models for aromatic infrared bands
}

\author{
Amit Pathak $\nmid$ and Shantanu Rastogi \\ Physics Department, D. D. U. Gorakhpur University, Gorakhpur - 273009, INDIA. \\ email: amitpathak1234@rediffmail.com
}

\begin{abstract}
Aromatic Infrared Bands (AIBs) show significant profile variations in different astrophysical environments. Theoretical IR data is used to develop emission models to understand these variations. A good match with the observed "7.7" $\mu \mathrm{m}$ feature from different regions is obtained.
\end{abstract}

Keywords. Astrochemistry, stars: circumstellar matter, infrared: ISM

\section{Introduction}

ISO data reveals important variations in peak positions and band shapes of the AIBs (ISO results 1996). Lack of extensive experimental data on PAHs enhances the importance of theoretical data that may be used to model AIBs. We report models of composite emission from different PAH size groups using calculated IR data (Pathak 2006).

\section{Emission models and astrophysical implications}

The emission spectrum for individual PAHs is computed using the absorption spectrum as input assuming canonical ensembles as suggested in the cascade emission model (Schutte et al. 1993, Cook \& Saykally 1998, Pech et al. 2002). Complete details of the emission modeling may be found elsewhere (Pathak \& Rastogi 2008). Obtained emission spectra of individual PAHs are co-added assuming equal number of species and are plotted using a Lorentzian profile with FWHM of $20 \mathrm{~cm}^{-1}$.

The model spectrum of neutrals is dominated by $\mathrm{C}-\mathrm{H}$ stretch and $\mathrm{C}-\mathrm{H}$ out-ofplane bend vibrations. The observed $\mathrm{C}-\mathrm{H}$ stretch $(3.3 \mu \mathrm{m})$ and $\mathrm{C}-\mathrm{H}$ out-of-plane bend $(11.2 \mu \mathrm{m})$ intensity ratio is a suitable parameter constraining the size of PAHs. The absorption intensities of $\mathrm{C}-\mathrm{H}$ stretch vibrations reduce drastically upon ionization while small intensity variations are observed for the $\mathrm{C}-\mathrm{H}$ wag modes. Among the AIBs the 6.2 and $7.7 \mu \mathrm{m}$ emission features are most intense. The $\mathrm{C}-\mathrm{C}$ stretch vibrations set up in ionized PAH molecules give rise to these bands. Profile variations observed in these bands are a direct measure of the background environments that excite the PAHs.

The spectral models are specifically used to correlate with the "7.7" $\mu \mathrm{m}$ band. The profiles of two different models are shown in Figure 1. Model 'a', comprising medium sized compact PAHs, has a stronger lower wavelength component as observed in UV-rich environments of $\mathrm{H}$ II regions and reflection nebulae and have been classified as $A^{\prime}$ profiles (Peeters et al. 2002). The spectral model comprising large PAHs has the $7.7 \mu \mathrm{m}$ band dominated by the higher wavelength component at $7.8 \mu \mathrm{m}\left(1285 \mathrm{~cm}^{-1}\right)$ with a shoulder at $7.6 \mu \mathrm{m}\left(1315 \mathrm{~cm}^{-1}\right)$. Such profiles conform with observations of relatively benign

$\dagger$ Present address: Inorganic and Physical Chemistry Department, I. I. Sc., Bangalore, India. 

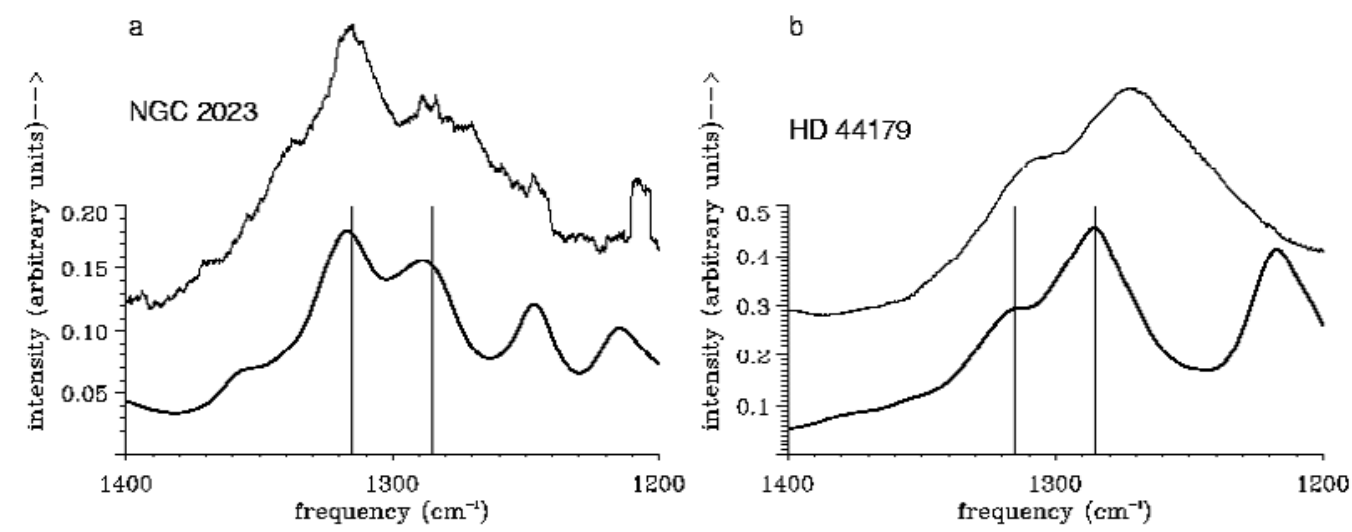

Figure 1. PAH cations emission model in the 1200 to $1400 \mathrm{~cm}^{-1}$ region compared with ISO spectra of PAHs with (a) less than $40 \mathrm{C}$ atoms in varying proportions, and (b) more than $40 \mathrm{C}$ atoms. The $7.7 \mu \mathrm{m}$ band lies within the two vertical lines.

Table 1. Band position and strength ratios of the two components of $7.7 \mu \mathrm{m}$ composite.

\begin{tabular}{|c|c|c|c|c|c|c|}
\hline PAH model & 7.6 & 7.8 & Diff. $\left(\mathrm{cm}^{-1}\right)^{1}$ & $\operatorname{Diff}_{. o b s}^{2}\left(\mathrm{~cm}^{-1}\right)$ & $I_{7.6} / I_{7.8}{ }^{1}$ & Obs $\mathrm{I}_{7.6} / \mathrm{I}_{7.8}{ }^{2}$ \\
\hline with $<40 \mathrm{C}$ atoms & 1347 & 1285 & 62 & $\sim 30$ & 1.06 & $\begin{array}{l}1.56 \text { (NGC 2023) } \\
1.35 \text { (Orion peak 2) } \\
1.20 \text { (IRAS 23133) }\end{array}$ \\
\hline with $>40 \mathrm{C}$ atoms & 1315 & 1285 & 30 & $\sim 45$ & 0.65 & $\begin{array}{l}0.64 \text { (NGC } 7027) \\
0.52 \text { (IRAS 17047) } \\
0.42 \text { (HD 44179) }\end{array}$ \\
\hline
\end{tabular}

${ }^{1}$ Difference of the band position and intensity ratio of the two components of the $7.7 \mu \mathrm{m}$ composite as calculated from the theoretical spectral models.;

${ }^{2}$ The observed results are from Peeters et al. (2002), Table 2.

astrophysical regions classified as $B^{\prime}$ profiles (Peeters et al. 2002). Intensity ratios of the two components of the $7.7 \mu \mathrm{m}$ complex and their wavenumber separation in the three models is presented in Table 1 .

\section{Conclusion}

The $7.8 \mu \mathrm{m}$ component, observed in benign regions, correlates with the model spectra of large PAH cations. The $7.6 \mu \mathrm{m}$ sub-feature dominant in UV rich regions matches the model spectra of medium sized PAH cations. This indicates formation of large PAHs in outflows of post-AGB stars that transform to medium sized ones in strong UV sources.

\section{References}

Cook, D. J. \& Saykally, R. J. 1998, ApJ, 493, 793

ISO Results 1996, $A \mathscr{E} A$ (Letters), 315, L26

Pathak, A. 2006, Ph.D. Thesis, D. D. U. Gorakhpur University, Gorakhpur (India)

Pathak, A. \& Rastogi, S. 2008, A\&SA, 485, 735

Pech, C., Joblin, C., \& Boissel, P. 2002, A\&A 388, 639

Peeters, E. et al. 2002, A\& A 390, 1089

Schutte, W. A., Tielens, A. G. G. M., \& Allamandola, L. J., 1993, ApJ, 415, 397 